\title{
Distorsiones Cognitivas y Estrategias de Afrontamiento en Jóvenes con Dependencia Emocional
}

\author{
María de la Villa Moral-Jiménez* y María Esther González-Sáez \\ Universidad de Oviedo
}

-Recibido: 08 - 09 - 2019 . Aceptado: 14 - 10 - 2019 . Avance Online: 31 - 10 - 2019

RESUMEN: Antecedentes: dadas las estrategias cognitivas diferenciales empleadas por los dependientes emocionales, en el presente estudio se pretende profundizar en el análisis de las distorsiones cognitivas y de las estrategias de afrontamiento más utilizadas por los jóvenes involucrados en una relación afectivo-dependiente. Método: han participado 241 adolescentes y jóvenes españoles con edades comprendidas entre los 16 y los 26 años ( $M=19.97$; $D T=2.51$ ). Se ha aplicado el Cuestionario de dependencia emocional (CDE), el Inventario de pensamientos automáticos (IPA) y el Cuestionario de afrontamiento COPE-28. Resultados: Se ha hallado que un $24.89 \%$ de los participantes presentan dependencia emocional. Estos jóvenes emplean estrategias de afrontamiento como el Apoyo social, la Religión, la Negación, la Autoinculpación, la Desconexión y el Desahogo, así como todas las distorsiones cognitivas evaluadas, excepto la Falacia de razón, en comparación con los jóvenes sin dependencia. Se ha comprobado que los chicos tienden a la Modificación de sus planes y a la Búsqueda de atención como aspectos representativos de la dependencia. No se han hallado diferencias en los niveles de dependencia emocional según el nivel de edad y el tiempo de relación. Conclusiones: los jóvenes con dependencia emocional presentan un perfil cognitivo característico con distorsiones cognitivas y estrategias de afrontamiento que cronifican su percepción diferencial de la relación afectivo-dependiente.

PALABRAS CAVE: Dependencia emocional, Distorsiones cognitivas, Estrategias de afrontamiento, Jóvenes, Perfil cognitivo.

Cognitive Distortions and Coping Strategies in Young People with Emotional Dependence

ABSTRACT: Background: Given the differential cognitive strategies used by emotional dependents in the present study, it is intended to deepen the analysis of cognitive distortions and the coping strategies most used by young people involved in an affective-dependent relationship. Method: 241 Spanish students, with ages between 16 and 26 years old $(M=19.97 ; S D=2.510)$, have participated in the research. The Emotional Dependence Questionnaire (CDE), the Automatic Thoughts Inventory (IPA) and the COPE-28 Coping Questionnaire have been applied. Results: It has been found that $24.89 \%$ of participants have emotional dependence. These young people employ coping strategies such as Social Support, Religion, Denial, Self-Inculpation, Disconnection and Relief, as well as all cognitive distortions evaluated, except the Fallacy of reason, compared to young people without dependence. It has been proven that boys tend to modify their plans and seek attention as representative aspects of dependence. No differences were found in levels of emotional dependence according to age level and relationship time. Conclusions: young people with emotional dependence have a characteristic cognitive profile with cognitive distortions and coping strategies that chronify their differential perception of the affectivedependent relationship.

KEY WORDS: Emotional dependence, Cognitive distortions, Coping strategies, Young people, Cognitive profile.

A nivel psicosocial, las vinculaciones interpersonales son fundamentales en el

*Correspondence: María de la Villa Moral-Jiménez.

Universidad de Oviedo.

CP: 33003, Oviedo, España.

E-mail:mvilla@uniovi.es

(C) 2020 Sociedad Universitaria de Investigación en Psicología y Salud. Publicado por Consejo General de Colegios Oficiales de Psicólogos, España. Este es un artículo Open Access
bajo la CC BY-NC-ND licencia (http://creativecommons.org/licencias/by-nc-nd/4.0/) proceso de construcción identitaria y en el desarrollo integral de la sociabilidad. Sin embargo, fuera de esos límites saludables pueden generarse vínculos patológicos y vulnerables (Lemos, Jaller, González, Díaz, y De la Ossa, 2012; Urbiola, Estévez, Iruarrizaga, y Jauregui, 2017). En este sentido, la 
Dependencia Emocional (DE) es descrita como un comportamiento desadaptativo contingente a una interrelación afectivo-dependiente, de modo que las personales emocionalmente dependientes intentan compensar su necesidad o deseo de afecto creando un apego patológico (Moral y Sirvent, 2008). La investigación en esta temática resulta de gran interés por las tasas de prevalencia y las implicaciones de semejante problemática a múltiples niveles. En cuanto a estudios realizados en España, Jiménez Burillo, Sangrador, Barrón y Yela (1995) encontraron que el $55 \%$ de su muestra reconocían ser dependientes emocionales. A su vez, Sirvent, Moral, Blanco y Palacios (2004) comprobaron que un $12 \%$ de la población general presentaba dependencia emocional, así como un $8.66 \%$ de forma severa, si bien en jóvenes universitarios aumenta la cifra a entre un $11.9 \%$ y un 25. 9\% (Sirvent y Moral, 2018), en base a unos criterios diagnósticos en los que se valora el craving y la abstinencia, el autoengaño, los conflictos identitarios con juegos de poder y los sentimientos negativos, entre otros factores patognomónicos.

La población joven es especialmente vulnerable a presentar en mayor grado dependencia emocional, teniendo en cuenta el hecho de que la prolongación artificial de la adolescencia en sociedades en crisis ha dado lugar a un descenso de la competencia emocional (Valle y Moral, 2018). Al valorarse la entrega incondicional a otra persona como uno de los factores principales de una relación, según Espinar, Zych y Rodríguez-Hidalgo (2015), los jóvenes potencian erróneamente la percepción de que cuanto más sufrimiento y más abducción emocional haya en la pareja, más se ama (Galán- Jiménez y Sánchez-ArmássCappello, 2018). Asimismo, se relacionan los antecedentes personales de carencias afectivas infantiles y el apego huidizo-temeroso con este trastorno, dado el modelo mental negativo tanto de sí mismo como de los demás y la necesidad constante de aprobación de los demás (Valle y Moral, 2018). A otro nivel, se ha constatado que los jóvenes victimizados psicológicamente y los agresores de este tipo de violencia presentan más dependencia emocional (Martín y Moral, 2019), incidiéndose en la disfuncionalidad de los vínculos afectivos asociada a una perpetuación generacional del ciclo de violencia. En consonancia con ello, en el meta-análisis de Gallego, Novo, Fariña y Arce (2019) se encontró que la probabilidad de desarrollar violencia de hijos a padres para los niños victimizados por sus padres aumentó un $71 \%$ en comparación con los no victimizados.

En cuanto a la relación entre la DE y el género, Bornstein (1995) realizó un metaanálisis donde halló mayor número de mujeres con dependencia emocional que hombres. Tales diferencias pueden interpretarse sobre la base de la desigual socialización de género, según la cual los roles de mayor subordinación son aceptados en mayor medida por mujeres (Ramiro-Sánchez, Ramiro, Bermúdez y BuelaCasal, 2018). En concreto, según Valor-Segura, Expósito y Moya (2014) las mujeres con alta dependencia de sus parejas se sienten más culpables que aquellas con baja dependencia en situaciones de alto conflicto. A su vez, Herrera, Herrera y Expósito (2018) hallaron que las respuestas de las mujeres con una alta carga emocional frente al acoso sexual (confrontación) son evaluadas negativamente. Por el contrario, en algunos estudios como el de Urbiola y Estévez (2015) se encuentra mayor número y mayores niveles de dependencia emocional en hombres, mientras que en otros estudios se constata que no hay diferencias basadas en el género (Martín y Moral, 2019; Urbiola et al., 2017).

Entre las principales características que identifican el perfil clínico y psicosocial de los dependientes emocionales destaca la subordinación a su pareja, la pérdida de su propia identidad, la posesividad, las escasas habilidades sociales, el apego excesivo, la poca autonomía, la reafirmación constante del amor hacia el sujeto del que se depende y la utilización de las relaciones para llenar su vacío emocional (González-Jiménez y Hernández-Romera, 2014; Lemos et al., 2012; Pradas y Perles, 2012) y, por tanto, experimentan insatisfacción constante y la recreación de sentimientos de soledad y abandono. De este modo, las personas emocionalmente dependientes desean la presencia continua de su pareja y su atención exclusiva, presentando, por tanto, temor ante las infidelidades y a que su pareja rompa la 
relación. Semejante anticipación de la ruptura se asocia a la puesta en marcha de conductas de vigilancia y control hacia sus pajeras con el empleo de juegos de poder poniendo en marcha tácticas para mantener la unión a toda costa (Estévez et al., 2018, Momeñe, Jauregui y Estévez, 2017; Valor-Segura, Expósito y Moya, 2014). Los dependientes emocionales asumen la culpa de lo que pase en la pareja, de ahí que perdonarán a la otra persona sin haber de por medio una resolución activa del problema (Valor-Segura et al., 2014). Desarrollan mecanismos de negación y autoengaño, de modo que se ha evidenciado que en población clínica, en concreto en drogodependientes y dependientes emocionales, se obtiene un perfil sindrómico de autoengaño más acusado que en población general (Moral y Sirvent, 2014). En este sentido, Sirvent (2007) explica que a causa de las ilusiones, ideas, creencias, expectativas - atribuciones que hacen presentar ceguera patológica y una gran vulnerabilidad emocional.

Para la comprensión de la dependencia emocional es imprescindible, según Lemos et al. (2012), conocer las creencias, esquemas desadaptativos y las distorsiones cognitivas, ya que estas crean unos perfiles que pueden dar lugar a situaciones de dependencia emocional. Los sujetos emocionalmente dependientes presentan un perfil cognitivo característico (Jaller y Lemos, 2009; Lemos et al., 2012) con un procesamiento de la información peculiar y con un gran número de distorsiones cognitivas, en opinión de Lemos, Londoño y Zapata (2007). De acuerdo con Beck, Rush, Shaw y Emery (2008) se podría definir la distorsión cognitiva como una serie de procesos por los cuales nuestro sistema cognitivo maneja la información, pero que no suelen estar ajustados a la realidad (Çayir y Kalkan, 2018). Es decir, representan creencias erróneas que el sujeto considera ciertas, las cuales afectan a su percepción y a su comportamiento (Beck, et al., 2008). Así, de acuerdo con Lemos et al. (2007), las distorsiones cognitivas están relacionadas con los esquemas disfuncionales, ya que estos son fortalecidos o mantenidos por los primeros. En este sentido, se considera que desarrollar esquemas desadaptativos pueden da lugar a la creación de relaciones tóxicas, trastornos o adicciones. De este modo, las personas dependientes emocionales utilizaban con mayor frecuencia distorsiones cognitivas como la Falacia de control, creyendo que la relación de pareja es su salvación y la distorsión llamada los Deberías, teniendo unas creencias muy rígidas (Lemos et al., 2007). También se alude a la Recompensa divina ya que piensan que la situación cambiará mágicamente, así como a la Falacia de razón al estimar que su opinión es la única y verdadera (Riofrío y Villegas, 2016).

Se estima que la dependencia emocional es causada y mantenida, entre otras razones, por la mala resolución de conflictos y la falta de estrategias de afrontamiento (Mayor, 2000). Así, se definirían las estrategias de afrontamiento como una serie de recursos y esfuerzos cognoscitivos y comportamentales, cuya función es resolver o reducir el problema cambiando las condiciones causantes de él, minimizar o eliminar la respuesta emocional o a cambiar la evaluación inicial que se había hecho del problema (Londoño et al., 2006; Moral de la Rubia, López, Díaz y Cienfuegos, 2011 ; Niño y Abaunza, 2015). Ciertamente, cada persona tiende a utilizar más unas estrategias que otras y esto forma un estilo de afrontamiento. En este sentido, Niño y Abaunza (2015) encontraron relación entre la dependencia emocional y la agresividad como estrategia de afrontamiento, si bien se constata una correlación negativa entre la dependencia emocional y la solución de problemas o la reevaluación positiva, lo cual se asocia a errores en las estrategias de afrontamiento y dificultades para una correcta solución a los problemas. De este modo, las personas con DE utilizan estrategias de afrontamiento evitativas con una tendencia a evitar la reevaluación cognitiva y desarrollando mecanismos de mixtificación y autoengaño (Martín y Moral, 2019). En suma, las personas con dependencia emocional emplean estrategias de afrontamiento desadapativas en virtud de las cuales se incrementan los desajustes en sus vínculos afectivo-dependientes.

\section{- OBJETIVOS E HIPÓTESIS}

Una vez fundamentado lo anterior, en este estudio se propone como objetivo general averiguar la relación entre la dependencia emocional, las distorsiones cognitivas realizadas y las estrategias de afrontamiento puestas 
en marcha por los sujetos. Como objetivos específicos, se propone determinar qué creencias y estrategias son más preponderantes en las personas con dependencia emocional. Asimismo, se comprobará si existe relación entre la dependencia emocional y el sexo, así como respecto a la edad y el tiempo que llevan en pareja.

Se plantean las siguientes hipótesis de investigación:

H1: Las principales distorsiones cognitivas que presentarán los sujetos dependientes emocionales respecto al resto será la tendencia a mantener reglas rígidas y exigentes (Debería) y a percibir que no tienen ningún control sobre los acontecimientos (Falacias de control).

H2: Se prevé un mayor afrontamiento pasivo en los dependientes emocionales, creyendo que la situación se solucionará sin hacer ellos nada al respecto, teniendo peso las estrategias de Aceptación, Autoinculpación, Desconexión, Negación y Uso de sustancias.

H3: Se supone que en los chicos se dará en mayor medida la búsqueda de atención como dimensión explicativa diferencial de la dependencia emocional y en las mujeres será la expresión afectiva y el miedo a la soledad.

H4: Se hipotetiza que la muestra de menor edad presentará mayor dependencia emocional y que el tiempo que llevan en la relación de pareja no estará relacionado con el nivel de dependencia.

\section{MÉTODO}

\section{- PARTICIPANTES}

La muestra, seleccionada de manera no probabilística, está compuesta por 241 participantes $(150$ mujeres, el $62.2 \%$ de la muestra), con edades comprendidas entre los 16 y los 26 años $(M=19.97$; DT = 2.51) de las Facultades de Psicología y de Derecho de la Universidad de Oviedo (Asturias, España) y de dos Centros de Enseñanza Obligatoria de
Cantabria (España). Respecto a la distribución por nivel educativo, el 23.2\% $(n=56)$ de los sujetos se encuentra estudiando Bachillerato y el $7.8 \%(n=185)$ una carrera universitaria.

En cuanto a las relaciones sentimentales, el $49 \%$ ( $n=118)$ de la muestra actualmente tiene una relación sentimental, de modo que se constata un amplio rango de variabilidad en cuanto al tiempo que llevan manteniéndola, entre 1 mes y 108 meses $(M=13.66$ meses; DT $=21.80)$. El $67,2 \%$ de los sujetos lleva en la relación entre un mes y un año, el 15\% entre uno y dos años y, por último, el 17.8\% mantiene la relación con su actual pareja entre 2 y 9 años.

\section{-INSTRUMENTOS DE MEDIDA}

Para desarrollar este estudio se han recabado diferentes datos sociodemográficos como sexo, edad, nivel de estudios, si actualmente mantienen una relación y desde hace cuánto tiempo. Además, se han analizado tres constructos: dependencia emocional, distorsiones cognitivas y estrategias de afrontamiento, medidos a través de diferentes cuestionarios, los cuales de describirán a continuación.

- Cuestionario de dependencia emocional (CDE) (Lemos y Londoño, 2006)

Este instrumento se creó con la finalidad de medir la dependencia emocional, tanto en hombres como en mujeres, de 16 a 55 años. Se construyó sobre el modelo de la terapia cognitiva de Beck, defendiendo que las personas emocionalmente dependientes presentan un perfil cognitivo característico. Aunque, en un principio, este cuestionario constaba de 66 ítems, la prueba quedó reducida a 23 ítems y seis factores. Cada ítem es valorado en una escala Likert de seis puntos con un rango de uno (Completamente falso de mí) a seis (Me describe perfectamente). Estos seis factores o subescalas son los siguientes: a) Factor 1, Ansiedad de separación: Detalla las expresiones emocionales de temor de los dependientes emocionales, que se generan ante un posible abandono de la pareja de 
estos; b) Factor 2, Expresión afectiva de pareja: Describe la necesidad que tiene el sujeto de recibir, por parte de su pareja, continuas expresiones de afecto para que de esa manera desaparezca la inseguridad; c) Factor 3, Modificación de planes: Explica el cambio de planes, comportamientos y actividades que la persona con dependencia suele hacer con el fin de agradar a su pareja, así como de estar próximo y compartir tiempo con ella; d) Factor 4, Miedo a la soledad: Detalla el sentimiento de pavor por no estar en una relación de pareja y sentir que nadie le ama o le quiere; e) Factor 5, Expresión de límite: Especifica las conductas límites e impulsivas de autoagresión que el dependiente emocional puede llevar a cabo ante la posibilidad de que su pareja le abandone y, finalmente, f) Factor 6, Búsqueda de atención: Este factor explica la tendencia que tienen los dependientes emocionales de ser el centro de atención de la vida de sus parejas, para mantener así la relación.

En el estudio de Lemos y Londoño (2006) el valor Alfa de Cronbach de las subescalas fluctúa entre .617 y .871 y el de la escala total fue de .927 , con una explicación de la varianza del $64.7 \%$. En cuanto al Alfa de Cronbach de la escala total en este estudio arroja un valor de .936 y los valores de las subescalas varían entre .886 y .609 .

- Inventario de pensamientos automáticos (IPA) (Ruiz y Luján, 1991)

Este inventario explora las distorsiones cognitivas, concebidas como representaciones mentales de nuestra experiencia en la vida asociadas a significados relacionados con una falsa imagen o interpretación. Los ítems se evalúan mediante una escala de tipo Likert que se valora de 0 (Nunca pienso eso) a 3 (Con mucha frecuencia lo pienso). La prueba consta de 45 ítems y de estos, tres ítems representan cada uno de los 15 tipos de distorsiones. Las distorsiones cognitivas son las siguientes: a) Abstracción selectiva: Seleccionar un solo aspecto de una situación, como en forma de túnel; b) Pensamiento polarizado: Valorar de manera extrema las situaciones, sin contar con aspectos intermedios; c) Sobregeneralización:
Predisposición a extraer una conclusión de un hecho general sin base alguna; d) Interpretación de pensamiento: Propensión a interpretar los sentimientos y propósitos de los demás, sin base suficiente; e) Visión catastrófica: Adelantarse, de manera calamitosa y grave, a los acontecimientos para los intereses personales; f) Personalización: Inclinación a relacionar o unir hechos del entorno o acontecimientos con uno mismo, sin tener datos o base suficiente; gl Falacia de control: Proclividad a creer que son responsables de todo lo que ocurre o todo lo contrario, tendencia a percibir que no tienen ningún control sobre los acontecimientos; h) Falacia de justicia: Disposición a considerar poco justo lo que no coincide con nuestros deseos o necesidades; i) Falacia de cambio: Creencia de que, ya que las cosas dependen de los demás, son ellos los que deben de cambiar primero; i) Razonamiento emocional: Creencia de que es verdadero, necesariamente, lo que la persona siente; k) Etiquetas globales: Propensión a generalizar unas pocas cualidades, en un juicio negativo global; l) Culpabilidad: Predisposición a asignar la responsabilidad de un acto a él mismo o a otros, sin base suficiente ni sopesando otros factores causantes; m) Debería: Tendencia a seguir y mantener reglas rígidas y exigentes, sobre como tienen que ser y suceder las cosas; n) Tener razón: Disposición a demostrar que el punto de vista de uno es el adecuado, y, finalmente, o) Falacia de recompensa divina: Se basa en la creencia de que la situación mejorará en el futuro, mágicamente, sin tener que buscar una solución al problema.

En cuanto a la fiabilidad del instrumento, Arriaga (2006) calculó la fiabilidad a través del método de las dos mitades de Guttman obteniendo un coeficiente de .933, para corregirlo por la fórmula de Spearman Brown, obteniéndose un valor para la escala total de .965 , lo que indica que es un instrumento fiable. En este estudio el valor del alfa de Cronbach es .943 .

- Cuestionario de afrontamiento COPE28 (Morán y Manga, 2009)

COPE-28 es la versión española del Brief COPE de Carver (1997), adaptada al español 
por Morán y Manga (2009) (documento sin publicar) y revisada por Morán, Landero y González (2010). Deriva de una versión más extensa realizada por el mismo autor y que fue adaptada al castellano por Crespo y Cruzado (1997). Es un inventario que evalúa la manera de afrontar y las respuestas ante el estrés y está compuesto por 28 ítems y 14 subescalas, que se responden en una escala ordinal tipo Likert de cuatro alternativas de respuesta desde 0 (No, en absoluto, nunca hago esto) a 3 (Mucho, hago siempre esto). Los 14 factores son los siguientes: a) Afrontamiento activo: Poner en marcha acciones y aumentar los esfuerzos por hacer desaparecer el estresor de manera directa; b) Planificación: Planear la dirección, los pasos, recursos y estrategias a poner en marcha para afrontar un problema; c) Apoyo instrumental o apoyo social: Obtener ayuda, consejos e información de personas que son competentes, cualificadas y capacitadas; d) Uso de apoyo emocional: Adquirir apoyo en forma de comprensión y simpatía; e) Auto-distracción: Centrarse en otras actividades, tratando de distraerse y no concentrarse en el problema; f) Desahogo: Manifestar los sentimientos de malestar para aliviarse, de la mano de un aumento de la conciencia de ese malestar; g) Desconexión conductual: Disminuir los esfuerzos para tratar con el problema y también para lograr los objetivos, con los cuales, se entorpece al problema; h) Reinterpretación positiva: Mirar el problema por el lado positivo y a partir de ello mejorar y crecer; i) Negación: Negar el problema existente y la realidad; i) Aceptación: Aceptar que es real y que el problema ocurre; k) Religión: Tender a acudir a la religión y a ser creyente en momentos de dificultades, aumentando la participación en actos religiosos; I) Uso de sustancias: Consumo de sustancias psicoactivas como alcohol, otras drogas o medicamentos, con la finalidad de sentirse mejor, aguantar y resistir la situación o problema; m) Humor: Bromear sobre el problema y, finalmente, n) Auto-inculpación: Culparse de la situación que acontece o de lo sucedido.

En el estudio de De la Cruz, Peña y Andreu (2015) el COPE-28, en su traducción al castellano y en su versión reducida, se obtuvo un coeficiente alfa de .60 para la escala total. En el presente estudio el alfa de Cronbach presenta un valor de .769 y el valor de las subescalas varía entre .953 y .590 .

\section{-PROCEDIMIENTO Y ANÁLISIS DE DATOS}

Para el estudio de recabó tanto muestra universitaria de los Grados de Psicología y de Derecho como de Bachiller manteniendo los pertinentes permisos y acuerdos de colaboración. Los cuestionarios se aplicaron en papel en sesiones de clase con el consentimiento del profesor que impartía la asignatura, de modo que se informó a los participantes de los fines del estudio incidiendo en el anonimato y obteniendo su consentimiento informado por escrito. La cumplimentación de los cuestionarios duró un tiempo estimado de 15-20 minutos.

Para el análisis de los resultados, por un lado, se utilizó una prueba $t$ de comparaciones de medias para muestras independientes para comprobar la existencia de diferencias en las distintas distorsiones cognitivas y en las estrategias de afrontamiento según el nivel de dependencia emocional. Asimismo, se empleó para analizar las diferencias en los factores de dependencia según el sexo, así como para analizar si existía mayor dependencia emocional según el nivel de estudios y el tiempo que llevan con sus parejas. Con la finalidad de determinar las relaciones entre las variables del estudio se realizó un análisis correlacional utilizando el coeficiente de correlación de Pearson entre los factores del cuestionario de dependencia emocional y los factores del inventario de distorsiones cognitivas, por un lado y por otro lado, otro análisis correlacional entre los factores de dependencia emocional con los factores del cuestionario de afrontamiento COPE28. Respecto al modelo explicativo de la dependencia emocional, se llevó a cabo una regresión binomial tanto con los factores del inventario de distorsiones cognitivas como con los factores de cuestionario de afrontamiento. Todos los análisis se han realizado a través del programa estadístico IBM SPSS Statistcs versión 25 . 


\section{RESULTADOS}

En primer lugar, se ha constatado que el $24.89 \%$ de los participantes presentan dependencia emocional. Respecto al empleo diferencial de las distorsiones cognitivas en jóvenes con y sin dependencia emocional se han hallado distorsiones cognitivas según la presencia de dependencia en todas las distorsiones, excepto en la Falacia de razón ( $p=$.070) (véase Tabla 1). Por tanto, puede decirse que las distorsiones más utilizadas por las personas con dependencia emocional serían Filtraje, Pensamiento polarizado, Sobregeneralización, Interpretación del pensamiento, Personalización, Falacia de control, Falacia de justicia, Falacia de cambio, Culpabilidad y los Debería ( $p<$ .000) mostrando en su mayoría un tamaño del efecto de .60, a excepción de las distorsiones Interpretación del pensamiento, los Debería y la Falacia de justicia con tamaños del efecto superiores, llegando al .93. Por su parte, Visión catastrófica ( $p=.025)$, Razonamiento emocional ( $p=.019)$, Etiquetas globales $(p=$
.040), y la Falacia de recompensa divina ( $p=$ .046) presentan un tamaño del efecto de entre 0.20 y 0.40 . Dada la diferente interpretación que se da a las categorías de Cohen para valorar la magnitud del tamaño del efecto, tomamos la Probabilidad de Superioridad del Tamaño del Efecto sobre el total de tamaños del efecto de Arce (Monteiro, Vázquez, Seijo, y Arce, 2018; Vilariño, Amado, Vázquez, y Arce, 2018). De este modo, se trata de evitar la relativización de las categorías de Cohen en pequeño, moderado y grande, mostrándose la magnitud de esa diferencia. Así, tal y como se expone en la Tabla 1, siendo en el primer factor Filtraje el tamaño del efecto $d=0.69$, al ser el valor $P S=.3758$, sería mayor que el $37.58 \%$ de todos los posibles de superioridad del grupo de no dependientes sobre dependientes".

De la misma manera, las estrategias de afrontamiento más utilizadas por las personas con dependencia emocional serían el Apoyo social ( $p=.006)$, la Religión $(p=.015)$, la Negación ( $p=$.000), la Autoinculpación $(p=.000)$, la Desconexión $(p=.000)$ y el Desahogo ( $p=.018)$, con tamaños del efecto

Tabla 1

Distorsiones cognitivas en función de la dependencia emocional

\begin{tabular}{|c|c|c|c|c|c|c|c|c|}
\hline \multirow{3}{*}{ Factores } & \multicolumn{4}{|c|}{ Tipo de Muestra } & \multirow{3}{*}{$t$} & \multirow{3}{*}{$p$} & \multicolumn{2}{|c|}{$\begin{array}{c}\text { Tamaño del } \\
\text { efecto }\end{array}$} \\
\hline & \multicolumn{2}{|c|}{$\begin{array}{c}\text { No dependientes } \\
(n=181)\end{array}$} & \multicolumn{2}{|c|}{ Dependientes $(n=60)$} & & & $d$ & PS \\
\hline & $M$ & $D T$ & $M$ & $D T$ & & & & \\
\hline Filtraje & 0.72 & 0.70 & 1.22 & 0.74 & -5.425 & .000 & -0.69 & .3758 \\
\hline Pensamiento polarizado & 0.49 & 0.63 & 0.90 & 0.76 & -4.185 & .000 & -0.59 & .3256 \\
\hline Sobregeneralización & 0.53 & 0.65 & 0.99 & 0.83 & -3.931 & .000 & -0.62 & .3400 \\
\hline Interpretación del pensamiento & 0.57 & 0.62 & 1.09 & 0.66 & -5.635 & .000 & -0.83 & .4380 \\
\hline Visión Catastrófica & 0.90 & 0.69 & 1.16 & 0.74 & -2.249 & .025 & -0.33 & .1820 \\
\hline Personalización & 0.57 & 0.51 & 0.92 & 0.57 & -4.498 & .000 & -0.65 & .3544 \\
\hline Falacia de Control & 0.56 & 0.53 & 0.92 & 0.60 & -4.438 & .000 & -0.64 & .3472 \\
\hline Falacia de Justicia & 0.57 & 0.69 & 1.09 & 0.79 & -4.904 & .000 & -0.71 & .3830 \\
\hline Razonamiento Emocional & 0.39 & 0.53 & 0.59 & 0.61 & -2.365 & .019 & -0.34 & .1896 \\
\hline Falacia de Cambio & 0.49 & 0.57 & 0.91 & 0.76 & -3.939 & .000 & -0.63 & .3400 \\
\hline Etiquetas globales & 1.01 & 0.64 & 1.22 & 0.70 & -2.062 & .040 & -0.30 & .1664 \\
\hline Culpabilidad & 0.46 & 0.46 & 0.78 & 0.58 & -3.925 & .000 & -0.62 & .3400 \\
\hline Los Debería & 0.88 & 0.69 & 1.54 & 0.74 & -6.422 & .000 & -0.94 & .4908 \\
\hline Falacia de Razón & 0.99 & 0.72 & 1.18 & 0.68 & -1.819 & .070 & -0.27 & .1428 \\
\hline
\end{tabular}


Tabla 2

Estrategias de afrontamiento en función de la dependencia emocional

\begin{tabular}{|c|c|c|c|c|c|c|c|c|}
\hline \multirow{3}{*}{ Factores } & \multicolumn{4}{|c|}{ Tipo de Muestra } & \multirow{3}{*}{$t$} & \multirow{3}{*}{$p$} & \multicolumn{2}{|c|}{ Tamaño del efecto } \\
\hline & \multicolumn{2}{|c|}{$\begin{array}{l}\text { No dependientes } \\
(n=181)\end{array}$} & \multicolumn{2}{|c|}{ Dependientes $(n=60)$} & & & $d$ & PS \\
\hline & $M$ & $D T$ & $M$ & $D T$ & & & & \\
\hline Afrontamiento activo & 2.11 & 0.60 & 2.02 & 0.61 & .95 & .342 & 0.14 & .080 \\
\hline Planificación & 1.88 & 0.76 & 1.74 & 0.80 & 1.24 & .214 & 0.18 & .103 \\
\hline Apoyo emocional & 1.74 & 0.83 & 1.85 & 0.80 & -.98 & .329 & -0.14 & .080 \\
\hline Apoyo social & 1.55 & 0.70 & 1.83 & 0.64 & -2.77 & .006 & -0.42 & .228 \\
\hline Religión & 0.28 & 0.61 & 0.56 & 0.81 & -2.48 & .015 & -0.39 & .221 \\
\hline Reevaluación positiva & 1.67 & 0.88 & 1.72 & 0.71 & -.53 & .600 & -0.07 & .040 \\
\hline Aceptación & 2.16 & 0.68 & 1.83 & 0.68 & 3.23 & .001 & 0.48 & .266 \\
\hline Negación & 0.32 & 0.60 & 0.73 & 0.76 & -3.83 & .000 & -0.60 & .333 \\
\hline Humor & 1.52 & 0.97 & 1.40 & 0.99 & .80 & .424 & .012 & .064 \\
\hline Autodistracción & 1.76 & 0.84 & 1.85 & 0.77 & -.76 & .451 & -0.12 & .064 \\
\hline Autoinculpación & 1.40 & 0.86 & 1.88 & 0.86 & -3.77 & .000 & -0.56 & .303 \\
\hline Desconexión & 0.44 & 0.53 & 0.80 & 0.65 & -3.98 & .000 & -0.58 & .318 \\
\hline Desahogo & 1.15 & 0.74 & 1.42 & 0.83 & -2.39 & .018 & -0.34 & .190 \\
\hline Uso de sustancias & 0.35 & 0.75 & 0.53 & 0.89 & -1.41 & .163 & -0.22 & .120 \\
\hline
\end{tabular}

entre $d=0.30$ y $d=0.60$ y PS que oscila entre el $33.3 \%$ y el $22.1 \%$ en las diferencias estadísticamente significativas, tal y como se puede apreciar en la Tabla 2, encontrando que la Aceptación ( $p=.001$; $d=0.48$ ) es una estrategia más utilizada por personas no dependientes emocionales.

Por otro lado, se ha confirmado que existen diferencias significativas en los factores de dependencia emocional según el sexo (véase Tabla 3), encontrando que los hombres muestran más Modificación de planes ( $p=$ .019; $d=0.30$ ) y Búsqueda de atención ( $p=$ $.002 ; d=0.42$ ) que las mujeres. Sin embargo, no se han encontrado diferencias significativas $\left(F_{1,241}=.704 ; p=n s\right)$ en dependencia emocional según los rangos de edad, ni tampoco respecto al tiempo que llevan los sujetos en la relación $\left(F_{1,241}=.471 ; p=\right.$ $.626)$.
Para contrastar la posible relación entre la dependencia emocional y las distorsiones cognitivas se usó un análisis correlacional. Se han obtenido correlaciones de Pearson superiores a .30 en las distorsiones cognitivas Filtraje, Sobregeneralización, Falacia de control, Falacia de justicia y Culpabilidad correlacionan con Ansiedad de separación, Expresión afectiva y Miedo a la soledad. Por su parte, la distorsión Interpretación del pensamiento correlaciona con Ansiedad de separación, Expresión afectiva, Modificación de planes y Miedo a la soledad. Las distorsiones los Deberías correlaciona además de con lo anterior con el factor de dependencia Expresión límite. Por otro lado, la distorsión cognitiva Falacia de cambio correlaciona con Expresión afectiva y Miedo a la soledad y, por último, la distorsión Pensamiento polarizado únicamente con Miedo a la soledad (véase Tabla 4). 
Tabla 3

Diferencias en dependencia emocional (CDE) en función del sexo

\begin{tabular}{|c|c|c|c|c|c|c|c|c|}
\hline \multirow{3}{*}{ Factores } & \multicolumn{4}{|c|}{ Tipo de Muestra } & \multirow{3}{*}{$t$} & \multirow{3}{*}{$p$} & \multicolumn{2}{|c|}{ Tamaño del efecto } \\
\hline & \multicolumn{2}{|c|}{ Varón $(n=181)$} & \multicolumn{2}{|c|}{ Mujer $(n=60)$} & & & $d$ & PS \\
\hline & $M$ & $D T$ & $M$ & $D T$ & & & & \\
\hline Ansiedad de separación & 2.07 & .941 & 2.00 & 1.01 & .533 & .594 & 0.071 & .0400 \\
\hline Expresión afectiva & 2.30 & 1.06 & 2.31 & 1.13 & -.083 & .934 & -0.010 & .0080 \\
\hline Modificación de planes & 1.94 & .981 & 1.66 & .87 & 2.352 & .019 & .0308 & .1742 \\
\hline Miedo a la soledad & 1.79 & .946 & 1.76 & 1.11 & .255 & .799 & 0.034 & .1600 \\
\hline Expresión límite & 1.38 & .587 & 1.28 & .64 & 1.219 & .224 & 0.163 & .0956 \\
\hline Búsqueda de atención & 3.00 & 1.09 & 2.53 & 1.11 & 3.192 & .002 & 0.425 & .2358 \\
\hline $\begin{array}{l}\text { Dependencia } \\
\text { emocional }\end{array}$ & 2.04 & .770 & 1.92 & .78 & 1.225 & .222 & 0.163 & .0956 \\
\hline Negación & 0.32 & 0.60 & 0.73 & 0.76 & -3.83 & .000 & -0.60 & .333 \\
\hline Humor & 1.52 & 0.97 & 1.40 & 0.99 & .80 & .424 & .012 & .064 \\
\hline Autodistracción & 1.76 & 0.84 & 1.85 & 0.77 & -.76 & .451 & -0.12 & .064 \\
\hline Autoinculpación & 1.40 & 0.86 & 1.88 & 0.86 & -3.77 & .000 & -0.56 & .303 \\
\hline Desconexión & 0.44 & 0.53 & 0.80 & 0.65 & -3.98 & .000 & -0.58 & .318 \\
\hline Desahogo & 1.15 & 0.74 & 1.42 & 0.83 & -2.39 & .018 & -0.34 & .190 \\
\hline Uso de sustancias & 0.35 & 0.75 & 0.53 & 0.89 & -1.41 & .163 & -0.22 & .120 \\
\hline
\end{tabular}

\section{Tabla 4}

Correlaciones de Pearson entre los factores del Inventario de pensamientos automáticos (IPA) y los factores del Cuestionario de dependencia emocional (CDE)

\begin{tabular}{|c|c|c|c|c|c|c|c|c|c|c|c|c|c|c|c|c|c|c|c|c|c|}
\hline & AS & EA & MP & MS & EL & BA & $\mathrm{F}$ & PP & SG & IP & VC & $P$ & FCon & FJ & RE & FCam & EG & C & D & FR & $\mathrm{RD}$ \\
\hline AS & - & & & & & & & & & & & & & & & & & & & & \\
\hline EA & $.741^{* *}$ & - & & & & & & & & & & & & & & & & & & & \\
\hline MP & $.633^{* *}$ & $.537^{* *}$ & - & & & & & & & & & & & & & & & & & & \\
\hline MS & $.569^{* *}$ & $.569^{* *}$ & $.477^{* *}$ & - & & & & & & & & & & & & & & & & & \\
\hline EL & $.535^{* *}$ & $.551^{* *}$ & $.496^{* *}$ & $.546^{* *}$ & - & & & & & & & & & & & & & & & & \\
\hline BA & $.601^{* *}$ & $.598^{* *}$ & $.436 * *$ & $.380^{* *}$ & $.320^{* *}$ & - & & & & & & & & & & & & & & & \\
\hline $\mathrm{F}$ & $.406^{* *}$ & $.395^{* *}$ & $.286^{* *}$ & $.494^{* *}$ & $.283^{* *}$ & $.245^{* *}$ & - & & & & & & & & & & & & & & \\
\hline PP & $.227^{* *}$ & $.250^{* *}$ & . $182^{2 *}$ & $.419^{* * *}$ & $.275^{* *}$ & .057 & $.636^{\prime \prime}$ & & & & & & & & & & & & & & \\
\hline SG & $.321^{* *}$ & $.304^{* \prime}$ & $.228^{* *}$ & $.454^{* *}$ & $.293^{* *}$ & .083 & $.683^{* \prime}$ & $.783^{* *}$ & - & & & & & & & & & & & & \\
\hline $\mathbb{P}$ & $.397^{* *}$ & $.344^{* *}$ & $.306^{* *}$ & $.351^{* *}$ & $.241^{* *}$ & $.238^{* *}$ & $.635^{* *}$ & $.609^{* *}$ & $.603^{* *}$ & - & & & & & & & & & & & \\
\hline vc & $.168^{* *}$ & $.183^{* *}$ & $.156^{*}$ & $.212^{* *}$ & $.132^{*}$ & .113 & $.497^{* *}$ & $.432^{* *}$ & $.414^{* *}$ & $.456^{* *}$ & - & & & & & & & & & & \\
\hline
\end{tabular}


Tabla 4 (Continuación)

Correlaciones de Pearson entre los factores del Inventario de pensamientos automáticos (IPA) y los factores del Cuestionario de dependencia emocional (CDE)

\begin{tabular}{|c|c|c|c|c|c|c|c|c|c|c|c|c|c|c|c|c|c|c|c|c|c|}
\hline & AS & EA & MP & MS & EL & BA & $\mathrm{F}$ & PP & SG & $\mathbb{P}$ & vc & $P$ & FCon & FJ & RE & FCam & $\mathrm{EG}$ & c & D & FR & $\mathrm{RD}$ \\
\hline$P$ & $.297^{* \prime}$ & $.342^{\prime \prime}$ & $.216^{\prime \prime}$ & $.356 "$ & $.252^{\prime \prime}$ & $.173^{* *}$ & $.541^{*}$ & $.628^{* \prime}$ & $.656 "$ & $.533^{* *}$ & $.460^{\circ *}$ & . & & & & & & & & & \\
\hline FCon & $.354^{* *}$ & $.330^{* *}$ & $.270^{\circ *}$ & $.337^{* *}$ & $.232^{* *}$ & $.249^{* *}$ & $.533^{* *}$ & .494"* & $.510^{\circ *}$ & .642"* & $.342^{\prime \prime}$ & $.534^{* *}$ & - & & & & & & & & \\
\hline FJ & $.337^{* *}$ & $.333^{* *}$ & $.214^{* *}$ & $.320^{* *}$ & $.222^{* *}$ & $.206^{* *}$ & $.581^{* *}$ & $.459^{* *}$ & $.430^{* *}$ & $.657^{* *}$ & $.405^{* *}$ & $.428^{* *}$ & $.644^{* *}$ & - & & & & & & & \\
\hline RE & $.221^{* *}$ & $.201^{* *}$ & $.151^{*}$ & $.243^{* \prime}$ & $.194^{* \prime}$ & .106 & $.590^{\circ *}$ & $.635^{* *}$ & $.581^{* \prime}$ & $.529^{* *}$ & $.527^{* *}$ & $.563^{* *}$ & $.420^{\circ *}$ & $.414^{* *}$ & - & & & & & & \\
\hline FCam & $.251^{*}$ & $.327^{* *}$ & $.287^{* *}$ & $.364^{* *}$ & $.230^{* *}$ & $.128^{*}$ & $.485^{\prime \prime}$ & .444"* & $.416^{* *}$ & $.517^{* *}$ & $.442^{\prime *}$ & $.444^{* *}$ & $.558^{* *}$ & $.565^{* *}$ & $.471^{* *}$ & - & & & & & \\
\hline EG & .110 & .109 & .066 & $.227^{* \prime}$ & .070 & .046 & $.418^{* \prime}$ & $.556 "$ & . & . $411^{* *}$ & $.470^{* *}$ & $.460^{\circ *}$ & $.400^{* *}$ & .407" & $.513^{* \prime}$ & $.370^{* \prime}$ & . & & & & \\
\hline c & $.327^{*}$ & $.310^{* *}$ & $.270^{* *}$ & $.392^{* \prime}$ & $.208^{* *}$ & $.198^{* *}$ & $.581^{* *}$ & $.621^{* *}$ & $.541^{* *}$ & $.626^{\prime \prime}$ & $.396^{* *}$ & $.567^{* *}$ & $.664^{*}$ & $.548^{* *}$ & $.490^{* *}$ & $.494^{* * *}$ & $477^{* *}$ & - & & & \\
\hline D & $.392^{* *}$ & $.424^{\prime \prime}$ & $.337^{* *}$ & $521^{* *}$ & $.369^{* *}$ & $.249^{* *}$ & $.653^{* *}$ & $.606^{* *}$ & $.563^{* *}$ & $.578^{* *}$ & $.504^{* *}$ & $.556^{\circ *}$ & $.554^{* *}$ & $.565^{* *}$ & $.577^{* *}$ & $.522^{* *}$ & $.460^{* *}$ & $.661^{* *}$ & - & & \\
\hline FR & $.142^{\circ}$ & $.150^{\circ}$ & .080 & .015 & -.004 & $.130^{*}$ & $.269^{\prime \prime}$ & $.154^{\circ}$ & .120 & $.417^{* *}$ & $.310^{0 *}$ & $.242^{2 *}$ & $.313^{* *}$ & $.449^{* *}$ & $.266^{* \prime \prime}$ & $.290^{\circ *}$ & $.341^{\prime \prime}$ & $.383^{\prime \prime}$ & $.312^{\prime \prime}$ & . & \\
\hline $\mathrm{RD}$ & $.228^{* \prime}$ & $.227^{* *}$ & .093 & .102 & .044 & $.225^{* *}$ & $.345^{* *}$ & .110 & .079 & .192"* & $.212^{* *}$ & .159 & $.274^{* *}$ & $.322^{* *}$ & .124 & $.225^{* *}$ & $.212^{* *}$ & $.310^{* *}$ & $.357^{* *}$ & .257" & - \\
\hline \multicolumn{22}{|c|}{$\begin{array}{l}\text { Nota: AS (Ansiedad de separación); EA (Expresión Afectiva); MP (Modificación de planes); MS (Miedo a la soledad); EL (Expresión Límite); BA (Búsqueda de atención); } \\
\text { F (Filtraje); PP (pensamiento polarizado); SG (Sobregeneralización); IP (interpretación del pensamiento);VC (Visión catastrófica); P (Personalización); FCon (Falacia } \\
\text { de control); FJ (Falacia de justicia); RE (Razonamiento emocional); FCam (Falacia de cambio); EG (Etiquetas globales); C (Culpabilidad); D (Deberías); FR (Falacia de } \\
\text { razón); RD (Recompensa divina). }\end{array}$} \\
\hline
\end{tabular}

Tabla 5

Correlaciones de Pearson entre los factores del Cuestionario de afrontamiento COPE-28 y los factores del Cuestionario de dependencia emocional (CDE).

\begin{tabular}{|c|c|c|c|c|c|c|c|c|c|c|c|c|c|c|c|c|c|c|c|c|}
\hline & AS & EA & MP & MS & EL & BA & AA & Pla & $\mathrm{AE}$ & AS & $R$ & RP & A & $\mathrm{N}$ & $\mathrm{H}$ & $A D$ & Al & Desc & Desa & US \\
\hline AS & & & & & & & & & & & & & & & & & & & & \\
\hline EA & $.741^{\prime \prime}$ & & & & & & & & & & & & & & & & & & & \\
\hline MP & $.633^{* \prime}$ & $.537^{* *}$ & & & & & & & & & & & & & & & & & & \\
\hline MS & $.569^{* *}$ & $.569^{\circ *}$ & $.477^{* *}$ & & & & & & & & & & & & & & & & & \\
\hline EL & $.535^{\prime \prime}$ & $.551^{* *}$ & $.496^{* *}$ & $.546^{\circ *}$ & & & & & & & & & & & & & & & & \\
\hline BA & $.601^{*}$ & $.598^{\circ *}$ & $.436^{* \prime}$ & $.380^{* *}$ & $.320^{\circ}$ & . & & & & & & & & & & & & & & \\
\hline AA & -.003 & -.074 & -.019 & $-.131^{*}$ & - & .032 & & & & & & & & & & & & & & \\
\hline Pla & -.038 & -.043 & -.034 & .031 & -031 & .025 & $.562^{* *}$ & & & & & & & & & & & & & \\
\hline $\mathrm{AE}$ & $.142^{*}$ & $.193^{* *}$ & .001 & .093 & -.004 & .181" & $.257^{* *}$ & $.210^{* *}$ & - & & & & & & & & & & & \\
\hline AS & $.267^{* \prime}$ & $.265^{* *}$ & .119 & $.158^{*}$ & .000 & $.228^{* *}$ & $.235^{* *}$ & $.143^{*}$ & $.666^{* *}$ & & & & & & & & & & & \\
\hline
\end{tabular}


Tabla 5 (Continuación)

Correlaciones de Pearson entre los factores del Cuestionario de afrontamiento COPE-28 y los factores del Cuestionario de dependencia emocional (CDE).

\begin{tabular}{|c|c|c|c|c|c|c|c|c|c|c|c|c|c|c|c|c|c|c|c|c|}
\hline & AS & EA & MP & MS & EL & BA & AA & Pla & $\mathrm{AE}$ & AS & $R$ & $\mathrm{RP}$ & A & $\mathrm{N}$ & H & $A D$ & $\mathrm{Al}$ & Desc & Desa & US \\
\hline $\mathrm{R}$ & $.215^{* *}$ & .187"* & $.209^{*}$ & . $174^{* *}$ & $.221^{*}$ & $.159^{*}$ & 110 & $.182^{* *}$ & -.033 & -.007 & & & & & & & & & & \\
\hline RP & -.011 & .040 & .050 & -.051 & .028 & $.130^{*}$ & $.317^{* *}$ & $.313^{* *}$ & $.261^{* *}$ & $.204^{* *}$ & $.131^{*}$ & & & & & & & & & \\
\hline A & $-.223^{* *}$ & $-.185^{* *}$ &.- .114 & $-.165^{*}$ & -.081 & -.095 & $.203^{* *}$ & $.230^{* *}$ & $.035^{* *}$ & .039 & .050 & $.274^{* * *}$ & & & & & & & & \\
\hline $\mathrm{N}$ & $.301^{* *}$ & $.298^{* *}$ & $.225^{* *}$ & $.290^{* *}$ & .149** & $201^{* *}$ & -.062 & .037 & $.178^{* *}$ & $.254^{* *}$ & .095 & .039 & $-.180^{*}$ & & & & & & & \\
\hline $\mathrm{H}$ & -.060 & .007 & -.124 & -.016 & -.018 & $.130^{\star}$ & .074 & $.229^{* *}$ & $.138^{*}$ & .102 & 100 & $.33^{* *}$ & $.254^{* *}$ & $.100^{* *}$ & . & & & & & \\
\hline$A D$ & .052 & .046 & .064 & .041 & .021 & .099 & $.167^{* *}$ & $.225^{* *}$ & $.199^{* *}$ & $.204^{* *}$ & .096 & $.152^{*}$ & $.203^{* *}$ & $.149^{*}$ & .065 & & & & & \\
\hline $\mathrm{Al}$ & $.271^{* *}$ & $.263^{* *}$ & $.178^{* *}$ & $.285^{* *}$ & $.206^{* *}$ & .125 & -.060 & .118 & -.028 & .053 & .095 & $-.168^{* *}$ & -.069 & $.293^{* *}$ & $.217^{* *}$ & $.148^{*}$ & & & & \\
\hline Desc & $.197^{* *}$ & $.288^{* *}$ & $.260^{* *}$ & $.308^{* *}$ & .199"* & .070 & $-.357^{* *}$ & - & .007 & .118 & $.133^{*}$ & $-.144^{*}$ & $-.147^{*}$ & $.402^{* *}$ & -.015 & $.149 *$ & $.275^{* *}$ & . & & \\
\hline Desa & $.200^{* *}$ & $.167^{* *}$ & .094 & .125 & .021 & .083 & $.220^{* *}$ & $.188^{* *}$ & $.237^{* *}$ & $.275^{* *}$ & .051 & .090 & 109 & $.226^{* *}$ & $.173^{* *}$ & 1ו1. & $.217^{* *}$ & .056 & & \\
\hline US & $.132^{\star}$ & $.138^{*}$ & 109 & 106 & .265 & .034 & $-.174^{* *}$ & $-.028^{*}$ & -.053 & -.006 & .070 & -.092 & $-.163^{*}$ & .182** & .070 & -.029 & $.211^{* *}$ & $.190^{* *}$ & -.011 & \\
\hline
\end{tabular}

Nota: AS (Ansiedad de separación); EA (Expresión Afectiva); MP (Modificación de planes); MS (Miedo a la soledad); EL (Expresión Límite); BA (Búsqueda de atención); AA (Afrontamiento activo); Pla (Planificación); AE (Apoyo emocional); AS (Apoyo social); R(Religión); RP ( Reevaluación Positiva); A (Aceptación); N (Negación); H (Humor); AD (Autodistracción); Al (Autoinculpación); Desc (Desconexión); Desa (Desahogo); US (Uso de sustancias).

${ }^{*} p<.05^{* *} p<.01$

En cuanto a las correlaciones entre las estrategias de afrontamiento y los factores de dependencia emocional (véase Tabla 5) únicamente correlacionan la estrategia Desconexión con el Miedo a la soledad y la estrategia Negación con Ansiedad de separación con valores superiores a 30.

Para hallar la relación entre la dependencia y las distorsiones cognitivas se utilizó una regresión logística binaria, según el cual se establece que las variables priorizadas en la ecuación serían los Debería, Interpretación del pensamiento y el Razonamiento emocional y los Debería (Tabla 6). Por otro lado, para las estrategias de afrontamiento según el nivel de dependencia (Tabla 7) la ecuación estaría compuesta por la Autoinculpación, la Aceptación, la Religión y el Apoyo social.

Tabla 6

Estrategias de afrontamiento en el modelo explicativo de la D.E.

\begin{tabular}{|c|c|c|c|c|c|c|c|c|}
\hline Variables & $B$ & $\begin{array}{c}\text { Error } \\
\text { estandar }\end{array}$ & Wald & gl & Sig. & Exp (B) & \multicolumn{2}{|c|}{95 \% C.I para Exp (B) } \\
\cline { 4 - 8 } Apoyo social & .658 & .231 & 8.114 & 1 & .004 & 1.931 & 1.228 \\
\hline Religión & .602 & .219 & 7.561 & 1 & .006 & 1.825 & 1.189 \\
\hline Aceptación & -.823 & .247 & 11.157 & 1 & .001 & .439 & .271 \\
\hline Autoinculpación & .601 & .194 & 9.637 & 1 & .002 & 1.824 & 1.248 \\
\hline
\end{tabular}


Tabla 7

Distorsiones cognitivas en el modelo de la D.E.

\begin{tabular}{|c|c|c|c|c|c|c|c|c|}
\hline \multirow{2}{*}{ Variables } & \multirow{2}{*}{$B$} & \multirow{2}{*}{$\begin{array}{c}\text { Error } \\
\text { estandar }\end{array}$} & \multirow{2}{*}{ Wald } & \multirow{2}{*}{ gl } & \multirow{2}{*}{ Sig. } & \multirow{2}{*}{$\operatorname{Exp}(B)$} & \multicolumn{2}{|c|}{$95 \%$ C.I para $\operatorname{Exp}(B)$} \\
\hline & & & & & & & Inferior & Superior \\
\hline $\begin{array}{l}\text { Interpretación del } \\
\text { pensamiento }\end{array}$ & .814 & .294 & 7.657 & 1 & .006 & 2.256 & 1.268 & 4.016 \\
\hline $\begin{array}{l}\text { Razonamiento } \\
\text { emocional }\end{array}$ & -.766 & .303 & 4.466 & 1 & .035 & .465 & .228 & .946 \\
\hline Los Debería & 1.171 & .286 & 16.792 & 1 & .000 & 3.224 & 1.842 & 5.643 \\
\hline
\end{tabular}

\section{DISCUSIÓN}

En esta investigación se ha comprobado que la mayoría de distorsiones cognitivas (tales como Filtraje, Pensamiento polarizado, Sobregeneralización, Interpretación del pensamiento, Visión catastrófica, Personalización, Falacia de control, Falacia de justicia, Razonamiento emocional, Falacia de cambio, Etiquetas globales, Culpabilidad, los Debería y la Falacia de recompensa divina) son utilizadas por las personas con dependencia emocional, a excepción de la Falacia de razón. En este sentido, Lemos etal. (2007) llegan a conclusiones parecidas encontrando diferencias significativas en todas las distorsiones excepto en Personalización, Etiquetas globales y Culpabilidad, siendo las más utilizadas por los dependientes emocionales los Debería y la Falacia de control. De la misma manera, Lemos et al. (2012) confirmaron que la distorsión Falacia de cambio es en la que más se incurre. En la misma línea, Riofrío y Villegas (2016) hallaron que en las quince distorsiones se presentan diferencias significativas concentrándose en el grupo de mayor dependencia emocional. Por lo que respecta a las estrategias de afrontamiento, los resultados de esta investigación coinciden con la hipótesis planteada al principio y con los hallazgos del estudio de Alonso (2017), quien llega a la conclusión de que las estrategias más utilizadas son Negación, Autoinculpación, Desconexión y Uso de sustancias, siendo esta última la única estrategia de afrontamiento no coincidente con los resultados del presente estudio. De este modo, Alonso (2017) también encontró que la estrategia de Aceptación tenía con la dependencia emocional una correlación negativa y, por tanto, las personas poco dependientes utilizaban más esta estrategia, iguales resultados a los que se ha llegado en este estudio.

En el análisis de dependencia emocional según el sexo se encontró que los hombres presentaban más Modificación de planes y Búsqueda de atención que las mujeres, en la misma dirección que los hallazgos obtenidos en el estudio de lturregui (2017) ya que los hombres puntuaban más en estas dos dimensiones. A este respecto, Lemos y Londoño (2006) obtuvieron diferencias significativas con relación al sexo en las diferentes subescalas, en el sentido de que las puntuaciones de las mujeres en Expresión afectiva de la pareja y Miedo a la soledad fueron mayores, mientras que los hombres reportaron puntuaciones superiores en Búsqueda de atención, al igual que lo hipotetizado en esta investigación. En estudios como los de Lemos et al. (2012) y Aponte (2015) no se encontraron diferencias significativas entre los niveles de dependencia emocional en función del sexo, al igual que en este estudio.

No se han hallado diferencias en los niveles de dependencia emocional según el nivel de estudios y según los rangos de edad. Sin embargo, en la investigación de Moral, García, Cuetos y Sirvent (2017) se obtienen resultados disímiles, encontrando mayores tasas de dependencia emocional en estudiantes de Enseñanza Secundaria Obligatoria que en universitarios. A su vez, Gamero y Salinas (2014) encuentran mayor porcentaje de dependientes emocionales en jóvenes de 17 a 20, que de 21 a 25. Por otro lado, en otros estudios con jóvenes se obtuvo que no existían diferencias significativas entre la dependencia emocional 
y la edad, al igual que la tendencia hallada en este estudio (véase Martín y Moral, 2019; Valle y Moral, 2018). En lo referente a la relación entre el tiempo que llevan los sujetos en la relación de pareja y la dependencia emocional se ha encontrado en este estudio que no hay diferencias, coincidiendo con la hipótesis inicial, y al igual que en la investigación de Gamero y Salinas (2014).

Finalmente, se plantean algunas de las limitaciones de este estudio que pueden estar influyendo en los resultados obtenidos. Por un lado, debería de tenerse en cuenta la posible influencia de variables internas propias de los sujetos de la muestra, por ejemplo el cansancio o el desinterés, que podrían estar afectando a la cumplimentación de los cuestionarios. Las respuestas de los sujetos también pueden estar influidas por la deseabilidad o reactividad al ser evaluados. Otras limitaciones son las relativas a la naturaleza transversal del estudio realizado que no permite extraer relaciones de influencia y la reducida muestra, así como los posibles sesgos que afecten a la representatividad de la misma.

Algunas de las mejoras que puede hacerse a este estudio serían conseguir un mayor tamaño muestral y más representativo, con participantes de diferentes comunidades autónomas, países y culturas, para proceder al análisis comparativo. Asimismo, como líneas futuras de investigación se propone controlar las variables externas, comentadas en las limitaciones, que puedan estar afectando a los resultados, pudiendo ser los cuestionarios cumplimentados en más de un momento temporal. También se recomienda añadir a este tipo de investigaciones otros métodos de evaluación, como entrevistas, para así disponer de mayor información siendo de interés para conocer con mayor profundidad la problemática, y crear así intervenciones ajustadas. Abogamos por la necesidad de potenciar la realización de estudios mediante los que se consolide la entidad gnoseológica de la dependencia emocional y se promueva su abordaje integral, dada la multicausalidad de esta problemática (Moral y Sirvent, 2014), así como por la relevancia de la implementación de programas de optimización de recursos socioemocionales en jóvenes (véase Eslami et al., 2015; Gilar-Corbi, Pozo y Castejón, 2019; Rodríguez, Orejudo, Celma y Cardoso, 2018; Rubiales, Russo, Panieva y González, 2018).

- Conflicto de intereses.

Los autores declaran no tener ningún conflicto de intereses.

\section{REFERENCIAS}

Alonso, R. (2017). Explicación de la dependencia emocional a partir de la autoestima y de las estrategias de afrontamiento. Madrid: Universidad Francisco de Vitoria.

Arriaga, N. (2006). Relación entre satisfacción familiar y distorsiones cognitivas en alumnos de psicología de la Universidad Cesar VallejoTrujillo (Tesis de Licenciatura). Trujillo, Perú: Universidad Cesar Vallejo-Trujillo.

Beck, A., Rush, J., Shaw, B., y Emery, G. (2008). Terapia cognitiva de la depresión. Bilbao: Desclée de Brouwer. Recuperado de https://www.edesclee.com/img/cms/ pdfs/9788433006264.pdf

Bornstein, R. F. (1995). Sex differences in objective and projective dependency tests: A meta-analytic review. Assessment, 2(4), 319-331. https://doi. org/10.1177/1073191195002004003

Carver, C. S. (1997). You want to measure coping but your protocol's too long: Consider the Brief COPE. International Journal of Behavioral Medicine, 4(1), 92-100. https:// doi.org/10.1207/s15327558iibm0401 6

Çayir, G. A., y Kalkan, M. (2018). The effect of interpersonal dependency tendency on interpersonal cognitive distortions on youths. Journal of Human Behavior in the Social Envionment, 28(6), 771-786. https://doi.org/ 10.1080/10911359.2018.1458681

Crespo, M., y Cruzado, J. A. (1997). La evaluación del afrontamiento: Adaptación española del cuestionario COPE con una muestra de estudiantes universitarios. Análisis y Modificación de Conducta, 23(92), 797-830.

Cruz de la, M. A., Peña, M. E., y Andreu, J. M. (2015). Creencias desadaptativas, estilos de afrontamiento y apoyo social como factores predictores de la vulnerabilidad psicopatológica en mujeres víctimas de 
agresión sexual. Clínica y Salud, 26(1), 33-39. https://doi.org/10.1016/i. clysa.2014.12.001

Eslami, A. A., Ghofranipour, F., Bonab, B. G., Zadeh, D. S., Shokravi, F. A., \& Tabatabaie, M. G. (2015). Evaluation of a school-based educational program to prevent adolescents' problem behaviors. Journal of Education and Health Promotion, 4(1), 30. https://doi. org/10.4103/2277-9531.154127

Espinar, E., Zych, I., y Rodríguez-Hidalgo, A. J. (2015). Ciberconducta y dependencia emocional en parejas jóvenes. Psychology, Society, \& Education, 7(1), 41-55. http:// dx.doi.org/10.25115/psye.v7i1.539

Estévez, A., Chávez-Vera, M. D., Momeñe, J., Olave, L., Vázquez, D., \& Iruarrizaga, I. (2018). The role of emotional dependence in the relationship between attachment and impulsive behavior. Anales de Psicología / Annals of Psychology, 34(3), 438-445. https:// doi.org/10.6018/analesps.34.3.313681

Galán-Jiménez, S. F., \& Sánchez-ArmássCappello, O. (2018). Psychometric properties of the dependent ideation scale. Anales de Psicología / Annals of Psychology, 34(3), 465-471. https://doi.org/10.6018/ analesps.34.3.318471

Gallego, R., Novo, M., Fariña, F., \& Arce, R. (2019). Child-to-parent Violence and Parentto-child Violence: A Meta-analytic Review. The European Journal of Psychology Applied to Legal Context, 17 (2), 51 -59. http://dx.doi. org/10.5093/ejpalc2019a4

Gamero, S. I., y Salinas, Y. S. (2014). Personalidad y dependencia emocional de la pareja, en universitarios. Arequipa: Universidad Católica de Santa María. Recuperado de https://docplayer.es/55564097-Universidadcatolica-de-santa-maria-facultad-de-cienciay-tecnologias-sociales-y-humanidadesprograma-profesional-de-psicologia.html

Gilar-Corbi, R., Pozo, T., y Castejón, J. L. (2019). Desarrollado la inteligencia emocional en educación superior: evaluación de la efectividad de un programa en tres países. Educación XXI: Revista de la facultad de Educación, 22(1), 161-187. https://doi. org/10.5944/educxx 1.19880

González-Jiménez, A. J., \& Hernández Romera, M. M. (2014). Emotional dependency based on the gender of Young adolescents in Almeria, Spain. Procedia-Social and Behavioral sciences, 6th International Conference on Intercultural Education "Education and Health: From a transcultural perspective", 132, 527-532. https://doi.org/10.1016/i. sbspro.2014.04.348

Herrera, M. C., Herrera, A., \& Expósito, F. (2018). To confront versus not to confront: Women's perception of sexual harassment. European Journal of Psychology Applied to Legal Context, 10, 1-7. https://doi.org/10.1016/i. ejpal.2017.04.002

Iturregui, C. R. (2017). Esquemas disfuncionales tempranos y dependencia emocional en estudiantes universitarios de Lima norte. Lima: Universidad peruana Cayetano Heredia. Recuperado de https://repositorio.upch.edu. pe/handle/upch/Esquemas IturreguiPaucar Cori

Jaller, C., y Lemos, M. (2009). Esquemas desadaptativos tempranos en estudiantes universitarios con dependencia emocional. Acta Colombiana de Psicología, 12(2), 77 83. Recuperado de www.scielo.org.co > pdf > acp v12n2a08.pdf

Jiménez Burillo, F., Sangrador, J. L., Barrón, P., y Yela, C. (1995). Análisis psicosocial sobre el comportamiento amoroso de los españoles. Madrid: C.I.S. Estudio n 2157.

Lemos, M., y Londoño, N. (2006). Construcción y validación del cuestionario de dependencia emocional en población Colombiana. Acta Colombiana de Psicología, 9 (2), 127-140. Recuperado de Disponible en: http://www. redalyc.org/articulo.oa? id $=79890212$

Lemos, M., Londoño, N. H., y Zapata, J. A. (2007). Distorsiones cognitivas en personas con dependencia emocional. Informes Psicológicos, 9(9), 55-69.

Lemos, M., Jaller, C., González, A. M., Díaz, Z. T., y De la Ossa, D. (2012). Perfil cognitivo de la dependencia emocional en estudiantes universitariosen Medellín, Colombia. Universitas Psicológica, $17(2), 395-404$. https://doi. 
org/10.1016/S0034-7450(14)60125-6

Londoño, N., Henao, G., Puerta, I., Posada, S., Arango, D., y Aguirre, D. (2006). Propiedades psicométricas y validación de la escala de estrategias de coping modificada (EEC-M) en una muestra colombiana. Universitas Psychologica, 5(2), 327-349. Recuperado de http://www.redalyc.org/articulo. oa? id $=64750210$

Martín, B., y Moral, M. V. (2019). Relación entre dependencia emocional y maltrato psicológico en forma de victimización y agresión en jóvenes. Revista Iberoamericana de Psicología y Salud, 10(2), 75-89. https://doi.org/10.23923/i. rips.2019.02.027

Mayor, I. (2000). ¿̇Por qué siempre nos enamoramos de quien no debemos? Madrid: Ediciones Librería Argentina.

Momeñe, J., Jauregui, P., y Estévez, A (2017). El papel predictor del abuso psicológico y la regulación emocional en la dependencia emocional. Behavioral Psychology / Psicología Conductual, 25(1), 65-78. Recuperado de https://www. behavioralpsycho. com/2018/10/04.Momeñe 25-1r.pdf

Monteiro, A., Vázquez, M. J., Seijo, D., y Arce, R. (2018). ¿̇Son los criterios de realidad válidos para clasificar y discernir entre memorias de hechos auto-experimentados y de eventos vistos en vídeo? Revista Iberoamericana de Psicología y Salud, 9(2), 149-160. https://doi. org/10.23923/i.rips.2018.02.020

Moral, M. V., y Sirvent, C (2008). Dependencias sentimentales $\mathrm{a}$ afectivas: etiología, clasificación y evaluación. Revista Española de Drogodependencia, 33(2), 150-167. Recuperado de https://www.aesed.com/ upload/files/vol-33/n-2/v33n2 2.pdf

Moral, M. V., y Sirvent, C (2014). Objetivos psicoterapéuticos e intervención integral en dependencias sentimentales. Revista Española de Drogodependencias, 39(3), 3856. Recuperado de https://www.aesed.com/ descargas/revistas

Moral, M. V., García, A., Cuetos, G., y Sirvent, C. (2017). Violencia en el noviazgo, dependencia emocional y autoestima en adolescentes y jóvenes españoles. Revista Iberoamericana de
Psicología y Salud, 8(2), 96-107. https://doi. org/10.23923/i.rips.2017.08.009

Moral de la Rubia, J., López, R., F., Díaz L., R., y Cienfuegos, Y. I. (2011). Diferencias de género en afrontamiento y violencia en la pareja. Revista CES Psicología, 4(2), 29-46. Recuperado de https://www.revistas.ces.edu.co/Inicio/Nol.4/

Núm.2/(2011)/MoraldelaRubia

Morán, C., Landero, R., \& González, M. T. (2010). COPE-28: A psychometric analysis of the Spanish version of the Brief COPE. Universitas Psychologica, 9(2), 543-552. Recuperado de https://www.researchgate.net/ publication/62440401 COPE-28

Niño, D. C., y Abaunza, N. (2015). Relación entre la dependencia emocional y afrontamiento en estudiantes universitarios. Revista Electrónica Psiconex: Psicología, Psicoanálisis y Conexiones, 7(10), 1-27. Recuperado de https:// aprendeenlinea.udea.edu.co/Psyconex/article/ view/23127

Pradas, E., y Perles, F. (2012). Resolución de conflictos de pareja en adolescentes, sexismo y dependencia emocional. Quaderns de Psicología, 14(1), 45-60. https://doi. org/10.5565/rev/qpsicologia. 1041

Ramiro-Sánchez, T., Ramiro, M. T., Bermúdez, M. P., \& Buela-Casal, G. (2018). Sexism in adolescent relationships: A systematic review. Psychosocial Intervention. 27(3), 123-132. https://doi.org/10.5093/pi2018a19

Riofrío, J. M., y Villegas, M. R. (2016). Distorsiones cognitivas según niveles de dependencia emocional en universitarios-Pimentel. Revista Paian, 7(1). Recuperado de revistas.uss.edu. pe > index.php > PAIAN, article > download

Rodríguez, C., Orejudo, S., Celma, L., y Cardoso, M. J. (2018). Mejora de las competencias socioemocionales en el aula, con jóvenes de Educación Secundaria, mediante el programa SEA. Electronic Journal of Research in Educational Psychology, 16(46), 681-701.

Rubiales, J., Russo, D., Panieva, J. P., y González, R. (2018). Revisión sistemática sobre los programas de Entrenamiento Socioemocional para niños y adolescentes de 6 a 18 años publicados entre 2011 y 2015. Revista Costarricense de Psicología, 37(2), 163-186. 
Recuperado de https://oaji.net , pdf , 32381545620666

Ruiz, J., y Luján, J. (1991). Manual de Psicoterapia Cognitiva. Inventario de pensamientos automáticos. Recuperado de http://www. psicologia -online.com/ESMUbeda/ Libros/ SentirseMejor/sentirse2.htm

Sirvent C., Moral M. V., Blanco, P. y Palacios L, (2004). Estudio sobre la interdependencia afectiva en la población general. Congreso de la Sociedad Española de Toxicomanías. Santiago de Compostela.

Sirvent, C. (2007). La mentira transformada. Paper presented at the $8^{\circ}$ Congreso Virtual de Psiquiatría Interpsiquis. Recuperado de https://www.researchgate.net, publication , 275022042

Sirvent, C., y Moral, M. V. (2018). Construcción y validación del inventario de relaciones interpersonales y dependencias sentimentales (IRIDS-100). Health and Addictions, 18(2), 35-47. https://dx.doi.org/10.21134/haai. v18i2.342

Urbiola, I., y Estévez, A. (2015). Dependencia emocional y esquemas desadaptativos tempranos en el noviazgo de adolescentes y jóvenes. Behavioral Psychology / Psicología Conductual, 23(3), 571-587. Recuperado de https://www.redalyc.org/ html/167/16750533007/
Urbiola, I., Estévez, A., Iruarrizaga, I., y Jauregui, P. (2017). Dependencia emocional en jóvenes: Relación con la sintomatología ansiosa y depresiva, autoestima y diferencias de género. Ansiedad y Estrés, 23(1), 6-11. http://dx.doi. org/10.1016/i.anyes.2016.11.003

Valle, L., y Moral, M. V. (2018). Dependencia emocional y estilo de apego adulto en las relaciones de noviazgo en jóvenes españoles. Revista Iberoamericana de Psicología y Salud, 9(1), 27-41. http://dx.doi.org/10.23923/i. rips.2018.01.013

Valor-Segura, I., Expósito, F., \& Moya, M. (2014). Gender, dependency and guilt in intimate relationship conflict among Spanish couples. Sex Roles, 70(1 1-12), 496-505. http://dx.doi. org/10.1007/s $11199-014-0351-2$

Vilariño, M., Amado, B. G., Vázquez, M. J., \& Arce, R. (2018). Psychological harm in women victims of intimate partner violence: Epidemiology and quantification of injury in mental health markers. Psychosocial Intervention, 27(3), 145 152. https://doi.org/10.5093/pi2018a23 